\title{
Obstetric implications of maternal chronic hepatitis B virus infection
}

\author{
Terence T. Lao* \\ Department of Obstetrics \& Gynaecology, The Chinese University of Hong Kong, Prince of Wales Hospital, Shatin, Hong Kong \\ SAR, China
}

*Correspondence: Terence T. Lao, Department of Obstetrics \& Gynaecology, The Chinese University of Hong Kong, Prince of Wales Hospital, Ngan Shing Street, Shatin, Hong Kong SAR, China. lao-tt@cuhk.edu.hk

Academic Editor: Elizabeth Ann L. Enninga, Mayo Clinic College of Medicine and Science, USA

Received: July 15, 2021 Accepted: September 17, 2021 Published: December 31, 2021

Cite this article: Lao TT. Obstetric implications of maternal chronic hepatitis B virus infection. Explor Med. 2021;2:468-82. https://doi.org/10.37349/emed.2021.00064

\begin{abstract}
Antenatal screening for hepatitis B surface antigen seropositivity is widely adopted to identify pregnant women with chronic hepatitis B virus (HBV) infection in order to target their newborn infants for combined passive-active neonatal immunization to prevent the maternal-to-child transmission of HBV. It is less certain whether the presence of chronic HBV infection in these largely asymptomatic women could impact their pregnancy outcome. There is now gathering information in the literature, though sometimes conflicting, on the obstetric implications of chronic HBV infection. The conflicting data is most probably related to confounding factors such as the immunological phase of chronic HBV infection, viral genotype and activity, presence of hepatic inflammation and other co-existing liver disorders such as non-alcoholic fatty liver disease, and coinfection with other virus such as hepatitis $C$ virus and micro-organisms, which are usually not examined, but which could have made significant influence on the occurrence of many of the pregnancy complications and adverse fetal and neonatal outcome. For pregnancy complications, the evidence suggests association with increased gestational diabetes mellitus, preterm birth, intrahepatic cholestasis of pregnancy, caesarean delivery, and postpartum haemorrhage, probably increased placental abruption and prelabour rupture of the membranes, and no effect or a reduction in the hypertensive disorders of pregnancy, especially preeclampsia. For perinatal outcome, there may be increased miscarriage and fetal malformations, and increase in both low birthweight and large-for-gestational age/macrosomic infants, as well as increased intrauterine fetal demise/stillbirth and fetal distress. However, most studies have not elaborated on the mechanisms or explanations of many of the adverse outcomes. Taken together, maternal chronic HBV infection increases the risk of adverse obstetric outcome overall, but further prospective studies are warranted to elucidate the reasons and mechanisms of, and with a view to mitigating, these adverse obstetric outcomes.
\end{abstract}

\section{Keywords}

Chronic hepatitis B virus infection, pregnancy, gestational diabetes mellitus, preterm birth, pre-eclampsia, intrahepatic cholestasis of pregnancy, birthweight, fetal loss 


\section{Introduction}

One of the routine antenatal screening tests is the assay for hepatitis B virus (HBV) surface antigen (HBsAg) serological status, in order to identify the mothers with chronic HBV infection who could pass the infection on to their offspring around the perinatal period, with maternal-to-child-transmission (MTCT) being the most important mode of infection especially in highly endemic areas $[1,2]$. The various aspects of the prevention of MTCT, including the use of immunoglobulin and antiviral agents during pregnancy, in addition to the effect of implementation of combined passive-active neonatal immunization, are extremely well covered in the literature so that there is little need for further repetition. However, the potential implications of maternal chronic HBV infection on obstetric outcome are often ignored. In recent decades, there have been cumulating reports on increased risk of adverse pregnancy and perinatal outcomes other than MTCT in pregnancies complicated by maternal HBV infection. This article provides a brief review of the reported effects of chronic HBV infection on pregnancy and perinatal outcome in order to enable clinicians to enhance their care for pregnant women with known or newly identified HBV infection, in order to optimize obstetric outcome in these women.

This narrative review includes the publications in the English language accessible through PubMed and Google Scholar in a systematic search using keywords which include hepatitis B, pregnancy, maternal, fetal, perinatal, and pregnancy outcome. The search was not restricted to certain geographical regions or populations, but restricted to publications which have identified the study group/exposed group as having HBV infection/HBV carriage, and/or HBsAg carriage/seropositivity, but excluded publications in which the form of hepatitis was not clearly stated, or when the reports described acute hepatitis. Owing to the marked heterogeneity in the methods of these studies, and the lack of information on many aspects of maternal HBV infection status, which is generally not investigated in an obstetric setting especially in older studies which utilized antenatal HBsAg seropositivity from routine screening as the only marker of exposure, meta-analysis would not be meaningful. The adjusted odds ratio (aOR)/relative risk provided in these studies, indicating adjustment for confounding variables which were however not standardized or uniform, are provided where appropriate.

\section{Background factors which could influence the effect of chronic HBV infection on obstetric outcome}

The detection of HBsAg is an indication of HBV infection, although HBsAg could be screened negative in occult infection. To simplify the following discussion, women in previous studies labelled as HBsAg seropositive, HBV carriers, chronic HBV infection and HBV infection would all be referred to as HBV carriers, and those with negative screening would be labelled as non-carriers, where appropriate.

The impact of chronic HBV infection on obstetric outcome depends to some extent on the circumstance of diagnosis of maternal infection. In mothers with symptomatic infection or known to have chronic infection prior to pregnancy, the clinical picture as well as the effect on pregnancy is related to the disease activity, the reason for diagnosis, the presence of complications and coinfection, and whether the mothers are on antiviral treatment or not. Hence the picture can vary from a relatively quiescent scenario to fulminating hepatitis and hepatic failure, which would imply that the pregnancy outcome could have varied from uncomplicated and successful outcome to early pregnancy loss or fetal demise in utero, and various other forms of adverse pregnancy and maternal outcome, including maternal death. However, in the great majority of mothers the diagnosis is made upon routine antenatal screening, with the mothers being almost always asymptomatic, and this is the group of pregnancies in which the impact of HBV infection often remains unclear or unknown, which will be addressed in this review. Some of these mothers may have slightly to moderately elevated hepatic enzymes, some of them could be hepatitis B envelope antigen (HBeAg) seropositive, and some may have high circulating HBV DNA levels, features which are largely related to the immunological phase of the infection in individual woman [3]. It is beyond the scope of this review to examine and describe all these factors in their possible interaction with the progression and outcome of pregnancy, as these factors have remained largely unexplored in the reported studies on the obstetric outcome of chronic HBV infection. Last but not 
least, the endemicity or prevalence of HBV infection in the study population also influences the likelihood of any demonstrable effect on obstetric outcome, as it would be unlikely that common obstetric complications would be influenced by chronic HBV infection in a population with low endemicity, or low prevalence in the study subjects, and vice versa. These confounding factors are listed in Table 1 . The following review and discussion are based on findings reported from studies on pregnant women with asymptomatic chronic HBV infection detected at routine antenatal screening. Any relevant or contributory roles of the additional factors such as elevated hepatic enzymes, HBeAg status, HBV DNA levels, and co-infection, will be alluded to where relevant literature is available.

Table 1. Factors which could influence the likelihood of any demonstrable effect of chronic HBV infection on obstetric outcome

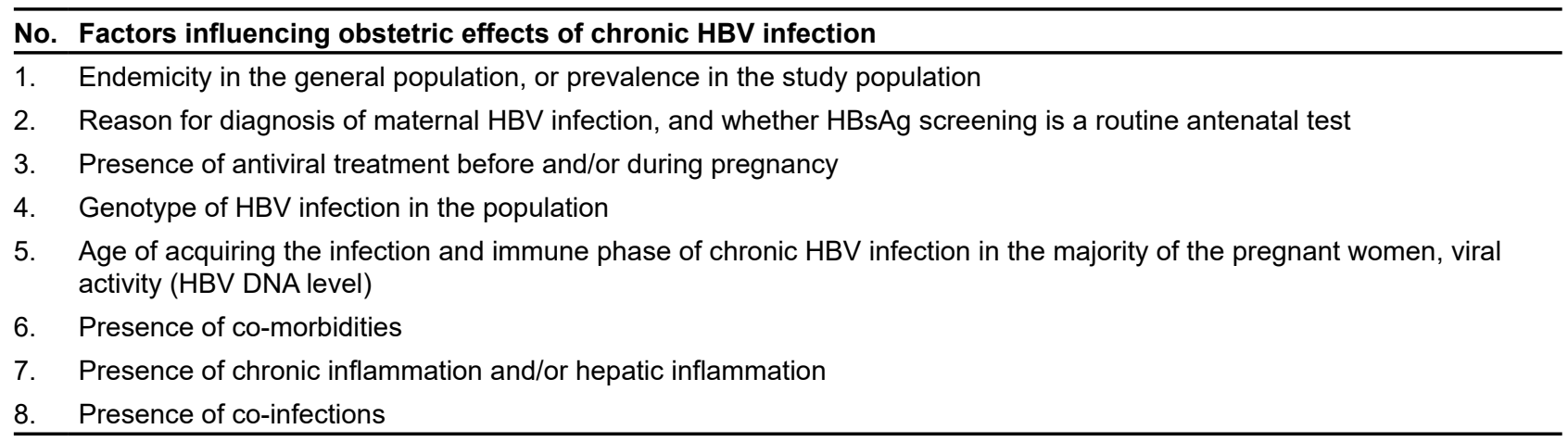

\section{Chronic HBV infection and pregnancy effects and complications}

The relationship between maternal chronic HBV infection with most of the major and common pregnancy complications has been studied, and this is summarized in Table 2. It is important to remember that the two primary and independent risk factors for adverse pregnancy outcome are advancing maternal age and nulliparity status. In endemic areas, both maternal age and parity status are shown to influence maternal HBsAg seropositivity at antenatal screening, the prevalence being highest in the third decade of life, and for women aged 25 to $<40$ years, multiparous mothers have significantly higher prevalence than nulliparous mothers for the same age groups [4]. The effect of age should not be underestimated because it has been demonstrated in young mothers aged $\leq 25$ years, who were born following the introduction of neonatal HBV immunization on offspring of HBsAg seropositive mothers, that the prevalence of HBsAg seropositivity at antenatal screening increased significantly from $1.0 \%$ in those aged $\leq 16$ years at delivery to $8.4 \%$ for those aged 25 years at delivery [5]. Indeed, it has been suggested that maternal chronic HBV infection could confer some form of reproductive advantage in its association with increasing maternal parity [6]. Thus, the interactions between HBV carrier status with age and parity in different study populations could have resulted in significant differences in outcome, so that these factors should be borne in mind in the interpretation of data reported from different centers and populations.

Table 2. Pregnancy complications in which demonstrable effects of chronic HBV infection have been reported

\begin{tabular}{lll}
\hline Pregnancy complication & Effect of HBV carrier status & References \\
\hline Gestational diabetes mellitus (GDM) & Increased & {$[7-17]$} \\
Pre-eclampsia (PE) & Increased superimposed PE & {$[12]$} \\
& Reduced & {$[22,24,27,39,40]$} \\
Pregnancy-induced hypertension (PIH)/ & Increased & {$[12,47,48]$} \\
gestational hypertension (GH) & Reduced & {$[22,24,27,39]$} \\
Intrahepatic cholestasis of pregnancy (ICP) & Increased & {$[26,27,50]$} \\
Preterm birth (PTB) & Increased & {$[12,13,19,23,51,53-57]$} \\
& Correlated with second trimester HBV DNA level & {$[48]$} \\
Prelabour rupture of membranes (PROM) & Increased in vaginal delivery & {$[27]$} \\
& Increased & {$[23,26]$} \\
\hline
\end{tabular}


Table 2. Pregnancy complications in which demonstrable effects of chronic HBV infection have been reported (continued)

\begin{tabular}{|c|c|c|}
\hline Pregnancy complication & Effect of HBV carrier status & References \\
\hline \multirow{3}{*}{$\begin{array}{l}\text { Antepartum haemorrhage (APH)/placental } \\
\text { abruption (PA) }\end{array}$} & Increased & {$[8,23]$} \\
\hline & Increased with hepatitis C virus co-infection & [19] \\
\hline & Increased in vaginal delivery & [27] \\
\hline Placenta praevia (PP) & No effect & {$[8,11,21,61]$} \\
\hline \multirow[t]{2}{*}{ Caesarean delivery $(C D)$} & Increased & {$[16,23,24,48]$} \\
\hline & Reduced & [19] \\
\hline Postpartum haemorrhage (PPH) & Increased & {$[24,27,65]$} \\
\hline
\end{tabular}

GDM

One of the pregnancy complications most frequently examined in HBV carriers is GDM, probably because GDM itself is among the most commonly encountered pregnancy complications. An association between maternal HBV carriers with GDM was first observed when HBV status was examined as a possible confounding factor in the relationship between maternal third trimester ferritin level at the time of the oral glucose tolerance test (OGTT) with the diagnosis of GDM [7]. This association was subsequently confirmed in a case-control study [8] and a cohort study in the same population [9]. This relationship was validated more recently in a repeat case-control study in the same population in which the OGTT was applied to both the study and control groups regardless of the presence or otherwise of established risk factors, when the HBV carriers exhibited a 77\% increased incidence of GDM [10]. This association has since been supported by a number of reports from different populations [11-16], and the positive association is unlikely to be influenced by the choice of the diagnostic criteria for the OGTT, since the $19.6 \%$ prevalence in the HBV carriers found in the case-control study referred to before [10] was similar to the $19.0 \%$ reported in the earlier case-control study [8], whereas the $12.3 \%$ prevalence in the control group was similar to the $10.2 \%$ in the non-carrier obstetric population [9] and 11.2\% in the matched high-risk non-carrier mothers [8]. In the latest report from China, increased GDM was confirmed with the aOR of 1.13 [16]. A meta-analysis on this issue has found an overall 47\% increased risk of GDM among HBV carriers [17]. This association is unrelated to HBV markers such as HBeAg status, viral load, or presence of circulating HBV DNA [10, 15], although a Thai study found 43.4\% increase in GDM when HBeAg was seropositive [13].

Nevertheless, a number of other studies [18-28] did not demonstrate such an association. The reasons for the conflicting findings have not been elucidated, but several factors could be involved. The most important factor is probably related to the method of screening and diagnosis of GDM. In the studies conducted in Hong Kong, there was either universal screening [7-9] or that OGTT was applied to everyone in both the study and control groups irrespective of the presence of risk factors [10]. It has been shown that between 34\% [29] and $54 \%$ [30] of the women with GDM diagnosed through universal screening were missed by risk factor-based screening. Thus, if the studies reporting negative findings did not apply universal screening, and that HBsAg seropositivity was not adopted as a risk factor for GDM, it was likely that there was an under-diagnosis of GDM among the HBV carriers. Another reason is that systemic inflammatory response is involved in the development of GDM [31,32], and early pregnancy elevation in hepatic alanine aminotransferase (ALT) is associated with subsequent GDM $[33,34]$. If the presence of low grade inflammation, especially hepatic inflammation, had not been examined in these studies, HBV carriers at risk of GDM would not have been screened and therefore the diagnosis could be missed. On the basis of the aforementioned studies, it would be prudent to include HBsAg seropositivity as a risk factor for GDM, especially in the populations with high prevalence of both chronic HBV infection and GDM, so that the occurrence of GDM would not be overlooked in case universal screening is not adopted as a routine antenatal test.

\section{PE}

PE belongs to the spectrum of pregnancy hypertensive disorders, which also included gestational hypertension (GH, also described as pregnancy-induced hypertension or PIH in some reports) [35]. Among this spectrum of disorders, the best defined is PE which refers to the onset of hypertension with proteinuria 
arising de novo, or superimposed on pre-existing hypertension. The diagnosis of GH/PIH and PE should be mutually exclusive, although some studies labelled both GH and PE together as PIH. There are many established risk factors for PE, including nulliparity status, advanced age ( $\geq 35$ years), high body mass index (BMI) at booking $\left(>25 \mathrm{~kg} / \mathrm{m}^{2}\right)$, and presence of significant medical history that included cardiovascular disease, renal and autoimmune disorders, and pre-GDM [36, 37]. Hence in the assessment of the relationship between maternal HBsAg seropositivity with PE, these confounding factors should also be taken into account.

There are conflicting findings in the literature. An increase in superimposed PE $1.1 \%$ versus $0.67 \%$ ) [12], as well as no association with PE [38], has both been reported, but the majority of studies reported a variable reduction in $\mathrm{PE}$, such as $0.73 \%$ versus $1.10 \%$ in one study [24], $0.22 \%$ versus $0.79 \%$ in another study [22], 29\% reduction in a third study [39], and a $20 \%$ reduction in a fourth study [27]. A meta-analysis which included 11 studies and 11,566 PE patients confirmed a 23\% reduction of PE [40]. It is proposed that the altered immune response in chronic HBV infection [41-43] is compatible with the maternal immune tolerance of the conceptus [39], thus reducing the risk of PE. Indeed, in mothers who develop PE, their immune responses include a shift in favor of T helper 1 (Th1) cell activity, activation of CD4+ and CD8 lymphocytes and deficiency of T regulatory cells, lower interleukin-10 (IL-10) production in peripheral blood mononuclear cells, and increased systemic inflammation with production of pro-inflammatory cytokines such as tumour necrosis factor- $\alpha$ (TNF- $\alpha$ ) [44-46], which resemble the immune clearance phase of chronic HBV infection. It is therefore possible that one important explanation of the conflicting findings is the phase of chronic HBV infection of the pregnant women in the study population, those who were trying to clear the virus could also manifest features of rejection of the conceptus hence predisposing to PE.

\section{PIH}

As explained in the preceding section, the condition described as PIH in some studies could be a mixture of different conditions. This may explain the reported increase in hypertensive disorders (15\% versus $8 \%$ ) in one study [47], $13 \%$ versus $2.89 \%$ in another study [12], and a 2.2 -fold increase in a third study [48]. However, the majority of studies found no difference in incidence $[8,11,19,23,38,49]$; or reduced GH $(0.97 \%$ versus $1.5 \%$ ) [24] and 17\% reduction [27], and reduced PIH (2.01\% versus 3.58\%) [22] and a 21\% reduction [39]. Bearing in mind the aforementioned confounding risk factors for hypertension in pregnancy [36, 37], as well as the relationship between chronic HBV infection with PE, it is very likely that maternal chronic HBV infection per se has no adverse effect on PIH.

\section{ICP}

This is a rare yet potentially very serious obstetric complication, but despite HBV being a hepatotropic virus, only a handful of studies conducted in China have examined this issue. In an earlier cohort study of 3,329 women, a 70\% increase in ICP was found among the $346 \mathrm{HBV}$ carriers as compared with the HBV negative women, and within the group of HBV carriers, ICP was more common among the HBeAg positive (aOR 2.96) then the HBeAg negative (aOR 1.52) women [26]. A recent report also supported this observation, with a much greater increase in ICP [odds ratio (OR)]3.4 found among the HBV carriers [27]. HBV infection and ICP together appeared synergistic, both together produced the highest rates for PROM, meconium-stained amniotic fluid, and CD compared with either complication alone [50]. These reports suggest that although a relatively uncommon complication, ICP could have been a hitherto overlooked event which could have resulted directly in, as well as contributing to, some of the adverse perinatal outcomes observed in some earlier studies.

\section{PTB}

In many reports, no distinction was made between spontaneous versus medically indicated PTB. Although threatened preterm labour was increased in HBV carrier mothers, it was not translated into increased PTB [8]. A later cohort study [51], and a meta-analysis of 6,781 women [52], also found no association between HBV carriers with preterm labour.

On the other hand, a majority of studies found increased PTB [12, 13, 19, 23, 53-55], which ranged from $1.3 \%$ in a study which also showed HBeAg seropositivity to increase PTB by $25 \%$ [13], to 5.2-fold in 
spontaneous PTB which was unrelated to the level of HBsAg or HBV DNA, or viraemia [53]. It is possible that viral activity plays a role here, as a Chinese study found no difference in overall PTB $(5.4 \%$ versus $5.0 \%$ ) between the 1,728 HBV carriers and 1,497 controls, but there was $18 \%$ increased PTB for each $\log _{10}$ copy/mL increase in second trimester HBV DNA [48]. A larger Chinese study with 489,965 women found seropositivity for HBsAg alone had $26 \%$ and $18 \%$ higher risk of PTB and early PTB ( $<34$ weeks gestation) respectively, while seropositivity for both $\mathrm{HBsAg}$ and $\mathrm{HBeAg}$ had $20 \%$ and $34 \%$ higher risk respectively [55]. Interestingly, a recent study found no increase in PTB overall, but when analyzed by mode of delivery, HBV carrier women who had vaginal delivery had a 14\% increase in PTB [27]. Increased PTB was confirmed in several meta-analyses which reported 2.36-fold increase [56], 26\% increase [51], and 16\% increase with HBV seropositivity alone and 21\% with both HBsAg and HBeAg seropositivity [57]. While the relationship between chronic HBV infection with increased PTB appears established, the extent of the effect could be quite variable due to differences in underlying or associated factors which have been explored in only a few studies.

One such factor is liver function. In a Chinese study, abnormal liver function test, half of which was related to non-alcoholic fatty liver disease, has turned out to be the independent risk factor for PTB rather than HBsAg seropositivity [58]. Furthermore, it is long established that preterm labour and PTB are largely attributed to the presence of genital tract and/or intra-amniotic infection [59], and it is possible that maternal chronic HBV infection predisposes to increased genital tract/intra-amniotic infection due to altered maternal immune response. As well, PTB is almost an inevitable outcome following preterm prelabour rupture of the membranes (PPROM), which was shown in a study that examined this complication specifically [12]. On the other hand, in countries with high endemicity for HBV infection such as China, PPROM is generally attributed to genital tract pathogens [60], and as genital tract or intrauterine infections were not specifically examined or excluded in the aforementioned studies, it is not possible to establish the independent role of chronic HBV infection in the occurrence of PTB with or without preceding PPROM. Thus, HBeAg status, hepatic inflammation, and possibly predisposition towards genital tract infection due to the immune modulation from chronic HBV infection, probably interact in different and varying ways to influence the occurrence of PTB.

\section{PROM}

The occurrence of PROM often occurs at term, but only a handful of studies have examined PROM specifically. A Chinese study found PROM to be increased by 38\% in HBsAg seropositive mothers [26], and an Israeli study found a 30\% increase [23], but it was not clarified if the cases of PROM all occurred at term or included those occurring preterm. In an Iranian study, although HBsAg seropositive mothers had higher incidence of PROM (6.89\% versus $2.44 \%$ ), this failed to reach statistical significance, probably due to the relatively small number of subjects, even though there was a significant 3.2-fold increase for PPROM [12]. Finally in a recent report, a 9\% reduction in PROM was found [27]. However, in the Chinese study comparing 346 HBsAg carriers with 2,983 non-carriers, PPROM was neither translated into increased PTB (19.8\% versus $16.6 \%)$ nor was it influenced by HBeAg status [26]. In these studies, other reported factors for PROM, including genital tract infections, were not examined or reported, and a definitive role of chronic HBV infection in the occurrence of PROM remains to be established.

\section{APH and PA}

The two major types of APH include PA and PP. In general, APH was increased by 2.18-fold with HBV alone [8], and 2.82-fold with HCV coinfection [19]. Where the type of APH was specified, HBsAg seropositivity was shown to increase PA by 4-fold [8] and 2-fold [23]. In a recent study which had also analyzed pregnancy outcome by mode of delivery, a $44 \%$ increased risk of PA was found in women with vaginal delivery [27]. However, there are a number of other confounding factors which influence the risk of PA, such as maternal smoking, and these were not examined or reported in the aforementioned reports. In the latest meta-analysis, no association with PA could be demonstrated [61]. 
Although there may be an association between HBsAg seropositivity with PA, no associated increase with PP was reported in the studies which examined this issue $[8,11,21]$. No association with PP was found in the latest meta-analysis [61]. Nevertheless, the preference of CD in some countries for the purported reason of reducing MTCT would inevitably increase the incidence of multiparous women with a scarred uterus, so that their risk of PP in subsequent pregnancies would likely be increased on account of their uterine scars [62].

\section{CD}

CD includes those performed as scheduled (elective) or as an emergency, but studies often did not distinguish between these two categories in their reports. In a US study comparing 814 HBV carriers with 296,218 non-carriers, the former group had 31\% reduction in $\mathrm{CD}$ [19]. On the other hand, a Hong Kong study on 9,526 women found more elective $\mathrm{CD}$ (13.8\% versus $12.9 \%)$ and less emergency $\mathrm{CD}(11.6 \%$ versus $15.5 \%)$, but the indications for CD were not provided [24]. An Israeli study on $749 \mathrm{HBV}$ (78.6\%) and/or HCV carriers found a $50 \%$ increase in overall CD [23]. Studies in China similarly documented increased CD by $70 \%$ in one study [48] and $12 \%$ in a more recent report [16]. It should be mentioned that in China, reducing or preventing MTCT is often taken as the indication for $\mathrm{CD}$, both on maternal request and obstetrician's recommendation [63]. But CD has been shown not to further reduce MTCT with timely neonatal immunoprophylaxis [64], so that elective $\mathrm{CD}$ is not indicated for this reason. Nevertheless, any associated pregnancy complications could conceivably increase the incidence of $\mathrm{CD}$, and future studies should adjust for pregnancy complications as confounders.

\section{PPH}

Few studies examined this outcome. A cohort study in Hong Kong has found increased PPH $(4.0 \%$ versus $2.7 \%$ ) although the mechanism for this association was not elaborated [24]. In another study from Hong Kong which examined the influence of maternal age on PPH, with maternal HBsAg status included as a potential confounding factor in the analysis, had unexpectedly found a $16 \%$ increase in $\mathrm{PPH}$, and again the mechanism of the increase was not further examined [65]. A recent report also demonstrated increased PPH (OR 1.16) [27]. It is possible that some of the increase in PPH could well be related to the associated increase in complications as well as CD, and this association should be clarified in further studies with adjustment for obstetric complications and interventions.

\section{HBsAg seropositivity and fetal/perinatal effects and complications}

Again, most adverse fetal and perinatal effects have been examined in relation to maternal HBV carrier status, as summarized in Table 3.

Table 3. Fetal/perinatal effects and complications in which demonstrable effects of chronic HBV infection have been reported

\begin{tabular}{lll}
\hline Fetal and perinatal effects and complications & Effect of maternal HBV carrier status & References \\
\hline Spontaneous miscarriage & Increased & {$[25,54,66]$} \\
Fetal congenital malformations & Increased & {$[23,50,54]$} \\
Intrauterine fetal demise (IUFD), stillbirth (SB), and & Increased & {$[12,23,54]$} \\
perinatal mortality & & {$[11,23,54,67]$} \\
Low birthweight (LBW) and small-for-gestational age (SGA) & Increased LBW infants & {$[13]$} \\
infants & Increased in HBeAg positive & {$[11,67]$} \\
& Increased SGA infants & {$[11,18,28,68]$} \\
& Reduced & {$[27]$} \\
Large-for-gestational age (LGA) and macrosomic infants & Increased birthweight in Oriental Chinese women $[24,47]$ \\
& Increased LGA/macrosomia & {$[12,48,68]$} \\
Fetal distress and birth asphyxia & Increased in HBeAg negative & {$[26]$}
\end{tabular}




\section{Spontaneous miscarriage}

An earlier study reported increased miscarriage among 264 women with viral hepatitis [54]. A later study found 71\% increased miscarriage when $513 \mathrm{HBV}$ carriers were compared with 20,491 controls [25]. As well, increased early abortion and overall abortion were also noted in HBV carrier women on in vitro fertilization (IVF) treatment, attributed to HBV infection of some embryos because HBV messenger ribonucleic acid (mRNA) fragments were detected in 13.2\% (5/38) and 5.6\% (1/18) of the cleavage embryos from the mothers or the fathers having HBV infection [66]. It is therefore possible that in women with HBV infection of their gametes or in their ovaries, there would be increased risk of spontaneous miscarriage, but as miscarriage is usually not included in the analysis of obstetric outcome, information on this issue is extremely limited. More detailed laboratory studies would be warranted to delineate the role of HBV infection of the gamete in miscarriage in these mothers.

\section{Fetal congenital malformations}

Fetal malformations, especially serious and lethal malformation, are collectively a cause for spontaneous miscarriage, and such data is usually not available in the studies on obstetric outcome. The fact that HBV mRNA fragments could be detected in $13.2 \%$ and $5.6 \%$ of the cleavage embryos from the mothers or the fathers having HBV infection [66] could be the underlying reason for the reported increased congenital malformations in the handful of studies which examined this issue [23, 50,54]. It is noteworthy that in one study [50], the rate of birth defect was $2 \%$ (1/50 cases) for maternal HBV infection, and increased to $16 \%(8 / 50$ cases $)$ among mothers with both HBV and ICP. However, the data should be interpreted with caution because in this study, there were only 50 cases of HBV infection, and 50 cases of ICP with HBV infection, compared with 50 controls, so that there could be some form of bias. In another study, the aOR for malformation was 1.4 [23]. However, no details on the types of malformations, or the organs/systems with malformation, were provided in these reports, and it remains unclear if there was an increase in overall structural malformations or some specific organs are involved in particular, and whether the increased malformations were mostly major, minor, or a mixture.

\section{IUFD, SB, and perinatal mortality}

Increased SB has been reported in an early study [54]. Subsequently other studies have also reported increased IUFD or SB, and perinatal mortality. A later study found markedly increased IUFD (5.56\% versus $0.44 \%$, OR 12.1$)$ and SB (2.89\% versus $0.44 \%$, OR 8.8$)$ between HBV carriers and non-carriers, but the distinction between IUFD and SB was not provided [12]. Another study found a significantly increased aOR of 1.8 for perinatal mortality [23]. The explanations for the increased IUFD, SB, and perinatal mortality were not provided in these studies, but factors which include increased congenital malformations, fetal hypoxaemia (see later discussion), and pregnancy complications such as PTB could all have contributed.

\section{LBW and SGA infants}

An increase in LBW $[11,23,54,67]$ and SGA $[11,67]$ infants have been reported. As LBW infants are often related to PTB, one of the explanations is likely to be the increased PTB in HBV carriers [51, 55-57]. Although another study found no increased LBW infants, HBeAg seropositivity was associated with $25.8 \%$ increase in LBW infants [13]. Nevertheless, the contribution of fetal growth restriction to LBW infants, and the potential mechanisms/explanations for the increased SGA infants, were not available.

\section{LGA and macrosomic infants}

A number of studies, examining this issue from different angles, suggest that maternal chronic HBV infection probably enhances fetal growth in utero. Some studies have found significant reduction in SGA infants, $20-25 \%$ in one study [11], 21\% in another [18], and $16 \%$ in a third [28]. Interestingly, one study found reduction in SGA infants (OR 0.68) only among women who had CD [27]. As well, increased birthweight was found in infants born to HBV carriers, although this was significant only in Oriental women [47]. Finally, increased macrosomic infants have been reported. In Iran, a 2.5-fold increase in macrosomic infants was found in a case-control study [12]. In Hong Kong, a cohort study confirmed an independent association 
with $15 \%$ and $11 \%$ increase in macrosomic and LGA infants respectively, and a $14 \%$ reduction in LBW infants [68]. Other Chinese studies similarly found higher mean birthweight [24], 68\% increase in macrosomic infants [48], and increased LGA infants (15.1\% versus $10.7 \%)$ which was confined to HBeAg negative women (aOR 1.91) [26].

The increase in LGA and macrosomic infants is intriguing, as the effect persisted despite controlled for the presence of GDM as a confounding factor [68]. The underlying mechanism could be related to that accounting for the reduction in PE $[21,22,39,40]$, in form of enhanced immune tolerance induced by chronic HBV infection, which becomes extended to the conceptus thus facilitating fetal growth as well. Another factor could be higher maternal ALT level, as non-GDM women delivering LGA infants had higher mean ALT levels and ALT level > 90th percentile (26 IU/L) was associated with a 4-fold increased LGA babies [69]. The enhanced immune tolerance and higher ALT level are most likely related to the phase of chronic HBV infection in the majority of these mothers.

\section{Fetal distress and birth asphyxia}

Increased fetal distress, including meconium stained amniotic fluid (MSAF), non-reassuring fetal heart rate (FHR) patterns, and low fifth minute Apgar score (AS), have been reported [22, 23, 48]. In one of these studies [48], maternal HBV infection was shown to be independently associated with fetal distress with aOR of 1.40, but the underlying reasons for fetal distress were not provided. In another study, there was $60 \%$ increased non-reassuring FHR patterns but 20\% lower incidence of MSAF [23]. A meta-analysis on 7,600 pregnant HBV carriers from 18 studies indicated an $80 \%$ increase in birth asphyxia [56]. The mechanisms of increased fetal distress probably reflect increased fetal hypoxaemia, which could help to explain the $80 \%$ increased perinatal mortality reported in one study [23], and the 12.1-fold increased IUFD and 8.8-fold increased SB in another study [12]. Irrespective of the mechanism, the increased IUFD, SB and fetal distress would call for proper fetal assessment and monitoring whenever fetal wellbeing is suspected to be jeopardized, and it is prudent to apply continuous intrapartum fetal monitoring for all pregnancies in HBV carriers.

\section{Postpartum and postnatal issues}

\section{Maternal postnatal depression}

It has been shown that non-pregnant asymptomatic HBV carriers were more likely to have psychiatric disorders than comparison subjects (30.2\% versus $11.6 \%)$, and had higher depression and anxiety scores and lower Global Assessment of Functioning scores [70]. Overall, up to half of patients with HBV infection have psychiatric morbidity [71]. Thus, it would be logical to anticipate increased psychiatric morbidity in HBV carriers. However, no studies has examined either antenatal and postnatal psychiatric or psychological status or disturbances, especially the risk of postnatal depression, in pregnant HBV carriers. Only one study examined pregnant HBV carriers and found higher levels of anxiety and stress in these mothers compared with healthy pregnant women [72]. However, it is unclear to what extent could such finding be influenced by the fact that these women only discovered their HBV carrier status at routine antenatal screening, as they were asymptomatic and unlike the patients who were aware and possibly symptomatic of their chronic HBV infection. Since postnatal depression could have serious and potential fatal consequences for the mother, prospective studies are warranted to elucidate the psychological status and psychiatric morbidity among mothers identified to have chronic HBV infection for the first time during pregnancy.

\section{Infant breast feeding}

Although breast feeding is often avoided to reduce MTCT, breast feeding did not increase MTCT [73], especially when timely combined passive-active neonatal immunoprophylaxis is administered routinely. The safety of breast feeding with regards to MTCT has been confirmed in a meta-analysis of 10 studies with 751 breast-fed and 873 non-breast-fed infants by assessing infant peripheral blood HBsAg or HBV DNA positivity at age 6-12 months, and antibody to hepatitis B surface antigen (anti-HBs) positivity at 6-12 months [74]. Therefore, breast feeding should be allowed with proper neonatal immunoprophylaxis. 


\section{Conclusions}

Taken together, the literature is highly suggestive of increase in certain adverse obstetric outcomes like GDM, PTB, ICP, PA, CD, and PPH, as well as fetal/perinatal morbidity and mortality, in pregnancies complicated by maternal HBV carriage. Paradoxically, HBV carriage also appears to confer some reproductive advantage in form of reduction in hypertension in pregnancy, especially PE, as well as enhanced fetal growth manifesting as reduction in LBW/SGA infants, and increase in birthweight as well as LGA and macrosomic infants. Indeed, a significant proportion of the studies found no or minimally increased adverse obstetric outcome in HBV carriers, and it was suggested that chronic HBV infection may have evolved to become synergistic with human reproduction in order to facilitate its transmission down successive human generations to perpetuate the infection in its primary host [28]. Most of the available studies on this topic are retrospective and are limited in the available information especially regarding the mechanisms or explanations involved in the association between chronic HBV infection with various reported outcomes. Clearly randomized controlled studies are ethically unacceptable and logistically unfeasible to explore and confirm these reported associations. Nevertheless, prospective observational studies with detailed planning, in depth investigations, and adjustment for the known confounding factors, should help to elucidate some of the mechanisms and clarify most of the reported associations. Meanwhile, it would appear prudent to include maternal HBV carriage as a risk factor for adverse pregnancy outcome so that these pregnancies could be flagged for enhanced maternal and fetal surveillance and monitoring in order to optimize obstetric outcome.

\section{Abbreviations}

ALT: alanine aminotransferase

aOR: adjusted odds ratio

APH: antepartum haemorrhage

BMI: body mass index

CD: caesarean delivery

GDM: gestational diabetes mellitus

GH: gestational hypertension

HBeAg: hepatitis B envelope antigen

HBsAg: hepatitis B virus surface antigen

HBV: hepatitis B virus

ICP: intrahepatic cholestasis of pregnancy

IUFD: intrauterine fetal demise

LBW: low birthweight

LGA: large-for-gestational age

MTCT: maternal-to-child-transmission

OGTT: oral glucose tolerance test

OR: odds ratio

PA: placental abruption

PE: pre-eclampsia

PIH: pregnancy-induced hypertension

PP: placenta praevia

PPH: postpartum haemorrhage

PPROM: preterm prelabour rupture of the membranes

PROM: prelabour rupture of membranes

PTB: preterm birth 
SB: stillbirth

SGA: small-for-gestational age

\section{Declarations}

Author contributions

The author contributes solely to this manuscript.

\section{Conflicts of interest}

The author declares that he has no conflicts of interest.

\section{Ethical approval}

Not applicable.

\section{Consent to participate}

Not applicable.

Consent to publication

Not applicable.

Availability of data and materials

Not applicable.

\section{Funding}

Not applicable.

\section{Copyright}

(C) The Author(s) 2021.

\section{References}

1. Beasley RP, Hwang LY, Lee GC, Lan CC, Roan CH, Huang FY, et al. Prevention of perinatally transmitted hepatitis B virus infections with hepatitis B immune globulin and hepatitis B vaccine. Lancet. 1983;2:1099-102.

2. Wong VC, Ip HM, Reesink HW, Lelie PN, Reerink-Brongers EE, Yeung CY, et al. Prevention of the HBsAg carrier state in newborn infants of mothers who are chronic carriers of HBsAg and HbeAg by administration of hepatitis-B vaccine and hepatitis-B immunoglobulin. Double-blind randomised placebo-controlled study. Lancet. 1984;1:921-6.

3. Liaw YF, Brunetto MR, Hadziyannis S. The natural history of chronic HBV infection and geographical differences. Antivir Ther. 2010;15 Suppl 3:25-33.

4. Suen SS, Lao TT, Sahota DS, Lau TK, Leung TY. Implications of the relationship between maternal age and parity with hepatitis B carrier status in a high endemicity area. J Viral Hepat. 2010;17:372-8.

5. Lao TT, Sahota DS, Law LW, Cheng YK, Leung TY. Age-specific prevalence of hepatitis B virus infection in young pregnant women, Hong Kong Special Administrative Region of China. Bull World Health Organ. 2014;92:782-9.

6. Lao TT, Sahota DS. Pregnancy and maternal chronic hepatitis B infection-evidence of reproductive advantage? Am J Reprod Immunol. 2017;77:e12667.

7. Lao TT, Tse KY, Chan LY, Tam KF, Ho LF. HBsAg carrier status and the association between gestational diabetes with increased serum ferritin concentration in Chinese women. Diabetes Care. 2003;26:3011-6. 
8. Tse KY, Ho LF, Lao T. The impact of maternal HBsAg carrier status on pregnancy outcomes: a case-control study. J Hepatol. 2005;43:771-5.

9. Lao TT, Chan BC, Leung WC, Ho LF, Tse KY. Maternal hepatitis B infection and gestational diabetes mellitus. J Hepatol. 2007;47:46-50.

10. Lao TT, Chung MK, Cheung TK, Law LW. Antenatal hepatitis B and increased risk of gestational diabetes mellitus-implications for obstetric care. J Infect. 2016;72:625-6.

11. Connell LE, Salihu HM, Salemi JL, August EM, Weldeselasse H, Mbah AK. Maternal hepatitis B and hepatitis C carrier status and perinatal outcomes. Liver Int. 2011;31:1163-70.

12. Saleh Gargari S, Hantoushzadeh S, Zendehdel N, Jamal A, Aghdam H. The association of maternal HBsAg carrier status and perinatal outcome. Hepat Mon. 2009;9:180-4.

13. Sirilert S, Traisrisilp K, Sirivatanapa P, Tongsong T. Pregnancy outcomes among chronic carriers of hepatitis B virus. Int J Gynaecol Obstet. 2014;126:106-10.

14. Tan J, Liu X, Mao X, Yu J, Chen M, Li Y, et al. HBsAg positivity during pregnancy and adverse maternal outcomes: a retrospective cohort analysis. J Viral Hepat. 2016;23:812-9.

15. Peng S, Wan Z, Lin X, Li X, Du Y. Maternal hepatitis B surface antigen carrier status increased the incidence of gestational diabetes mellitus. BMC Infect Dis. 2019;19:147.

16. Zhao Y, Chen YL, Song HQ, Huang PY, Wang LY, Liu W, et al. Effects of maternal hepatitis B surface antigen positive status on the pregnancy outcomes: a retrospective study in Xiamen, China, 2011-2018. PLoS One. 2020;15:e0229732.

17. Tan J, Mao X, Zhang G, Wang W, Pan T, Liu X, et al. Hepatitis B surface antigen positivity during pregnancy and risk of gestational diabetes mellitus: a systematic review and meta-analysis. J Viral Hepat. 2018;25:1372-83.

18. Bajema KL, Stankiewicz Karita HC, Tenforde MW, Hawes SE, Heffron R. Maternal hepatitis B infection and pregnancy outcomes in the United States: a population-based cohort study. Open Forum Infect Dis. 2018;5:ofy134.

19. Reddick KL, Jhaveri R, Gandhi M, James AH, Swamy GK. Pregnancy outcomes associated with viral hepatitis. J Viral Hepat. 2011;18:e394-8.

20. Stokkeland K, Ludvigsson JF, Hultcrantz R, Ekbom A, Höijer J, Bottai M, et al. Pregnancy outcome in more than 5000 births to women with viral hepatitis: a population-based cohort study in Sweden. Eur J Epidemiol. 2017;32:617-25.

21. Wong S, Chan LY, Yu V, Ho L. Hepatitis B carrier and perinatal outcome in singleton pregnancy. Am J Perinatol. 1999;16:485-8.

22. To WW, Cheung W, Mok KM. Hepatitis B surface antigen carrier status and its correlation to gestational hypertension. Aust N Z J Obstet Gynaecol. 2003;43:119-22.

23. Safir A, Levy A, Sikuler E, Sheiner E. Maternal hepatitis B virus or hepatitis C virus carrier status as an independent risk factor for adverse perinatal outcome. Liver Int. 2010;30:765-70.

24. Mak SL, Leung KY. Hepatitis B carriers in Hong Kong: prevalence and pregnancy outcomes. Hong Kong J Gynaecol Obstet Midwifery. 2013;13:67-73.

25. Cui AM, Cheng XY, Shao JG, Li HB, Wang XL, Shen Y, et al. Maternal hepatitis B virus carrier status and pregnancy outcomes: a prospective cohort study. BMC Pregnancy Childbirth. 2016;16:87.

26. Cai Q, Liu H, Han W, Liu L, Xu Y, He Y, et al. Maternal HBsAg carriers and adverse pregnancy outcomes: a hospital-based prospective cohort analysis. J Viral Hepat. 2019;26:1011-8.

27. Zhang Y, Chen J, Liao T, Chen S, Yan J, Lin X. Maternal HBsAg carriers and pregnancy outcomes: a retrospective cohort analysis of 85,190 pregnancies. BMC Pregnancy Childbirth. 2020;20:724.

28. Sun Q Lao TT, Du M, Xie M, Sun Y, Bai B, et al. Chronic maternal hepatitis B virus infection and pregnancy outcome-a single center study in Kunming, China. BMC Infect Dis. 2021;21:253. 
29. Cosson E, Benchimol M, Carbillon L, Pharisien I, Pariés J, Valensi P, et al. Universal rather than selective screening for gestational diabetes mellitus may improve fetal outcomes. Diabetes Metab. 2006;32:140-6.

30. Griffin ME, Coffey M, Johnson H, Scanlon P, Foley M, Stronge J, et al. Universal vs. risk factor-based screening for gestational diabetes mellitus: detection rates, gestation at diagnosis and outcome. Diabet Med. 2000;17:26-32.

31. Qiu C, Sorensen TK, Luthy DA, Williams MA. A prospective study of maternal serum C-reactive protein (CRP) concentrations and risk of gestational diabetes mellitus. Paediatr Perinat Epidemiol. 2004;18:377-84.

32. Lao TT, Tam KF. Maternal serum ferritin and gestational impaired glucose tolerance. Diabetes Care. 1997;20:1368-9.

33. Yarrington CD, Cantonwine DE, Seely EW, McElrath TF, Zera CA. The association of alanine aminotransferase in early pregnancy with gestational diabetes. Metab Syndr Relat Disord. 2016;14:254-8.

34. Leng J, Zhang C, Wang P, Li N, Li W, Liu H, et al. Plasma levels of alanine aminotransferase in the first trimester identify high risk Chinese women for gestational diabetes. Sci Rep. 2016;6:27291.

35. Hutcheon JA, Lisonkova S, Joseph KS. Epidemiology of pre-eclampsia and the other hypertensive disorders of pregnancy. Best Practice Res Clin Obstet Gynaecol. 2011;25:391-403.

36. Duckitt K, Harrington D. Risk factors for pre-eclampsia at antenatal booking: systematic review of controlled studies. BMJ. 2005;330:565.

37. Bellamy L, Casas JP, Hingorani AD, Williams DJ. Pre-eclampsia and risk of cardiovascular disease and cancer in later life: systematic review and meta-analysis. BMJ. 2007;335:974.

38. Huang X, Tan H, Li X, Zhou S, Wen SW, Luo M. Maternal chronic HBV infection would not increase the risk of pregnancy-induced hypertension-results from pregnancy cohort in Liuyang rural China. PLoS One. 2014;9:e114248.

39. Lao TT, Sahota DS, Cheng YK, Law LW, Leung TY. Maternal hepatitis B surface antigen status and incidence of pre-eclampsia. J Viral Hepat. 2013;20:343-9.

40. Huang QT, Chen JH, Zhong M, Hang LL, Wei SS, Yu YH. Chronic hepatitis B infection is associated with decreased risk of preeclampsia: a meta-analysis of observational studies. Cell Physiol Biochem. 2016;38:1860-8.

41. Stoop JN, van der Molen RG, Baan CC, van der Laan LJ, Kuipers EJ, Kusters JG, et al. Regulatory T cells contribute to the impaired immune response in patients with chronic hepatitis B virus infection. Hepatology. 2005;41:771-8.

42. Bertoletti A, Gehring AJ. The immune response during hepatitis B virus infection. J Gen Virol. 2006;87:1439-49.

43. Peng G, Li S, Wu W, Sun Z, Chen Y, Chen Z. Circulating CD4+ CD25+ regulatory T cells correlate with chronic hepatitis B infection. Immunology. 2008;123:57-65.

44. Jonsson Y, Rubèr M, Matthiesen L, Berg G, Nieminen K, Sharma S, et al. Cytokine mapping of sera from women with preeclampsia and normal pregnancies. J Reprod Immunol. 2006;70:83-91.

45. Laresgoiti-Servitje E, Gómez-López N, Olson DM. An immunological insight into the origins of pre-eclampsia. Hum Reprod Update. 2010;16:510-24.

46. Pinheiro MB, Martins-Filho OA, Mota AP, Alpoim PN, Godoi LC, Silveira AC, et al. Severe preeclampsia goes along with a cytokine network disturbance towards a systemic inflammatory state. Cytokine. 2013;62:165-73.

47. Pastorek JG 2nd, Miller JM Jr, Summers PR. The effect of hepatitis B antigenemia on pregnancy outcome. Am J Obstet Gynecol. 1988;158:486-9. 
48. Wan Z, Zhou A, Zhu H, Lin X, Hu D, Peng S, et al. Maternal hepatitis B virus infection and pregnancy outcomes: a hospital-based case-control study in Wuhan, China. J Clin Gastroenterol. 2018;52:73-8.

49. Lobstein S, Faber R, Tillmann HL. Prevalence of hepatitis B among pregnant women and its impact on pregnancy and newborn complications at a tertiary hospital in the eastern part of Germany. Digestion. 2011;83:76-82.

50. Hu Y, Ding YL, Yu L. The impact of intrahepatic cholestasis of pregnancy with hepatitis B virus infection on perinatal outcomes. Ther Clin Risk Manag. 2014;10:381-5.

51. Cui AM, Shao JG, Li HB, Shen Y, Chen ZX, Zhang S, et al. Association of chronic hepatitis B infection with preterm birth: our experience and meta-analysis. J Perinat Med. 2017;45:933-40.

52. Huang QT, Wei SS, Zhong M, Hang LL, Xu YY, Cai GX, et al. Chronic hepatitis B infection and risk of preterm labor: a meta-analysis of observational studies. J Clin Virol. 2014;61:3-8.

53. Elefsiniotis I, Tsoumakas K, Vezali E, Glynou I, Drakoulis N, Saroglou G. Spontaneous preterm birth in women with chronic hepatitis B virus infection. Int J Gynaecol Obstet. 2010;110:241-4.

54. Pavel A, Tîrşia E, Maior E, Cristea A. Detrimental effects of hepatitis B virus infection on the development of the product of conception. Virologie. 1983;34:35-40.

55. Liu J, Zhang S, Liu M, Wang Q Shen H, Zhang Y. Maternal pre-pregnancy infection with hepatitis B virus and the risk of preterm birth: a population-based cohort study. Lancet Glob Health. 2017;5:e624-32.

56. Luo L, Wu J, Qu Y, Li J, Pan L, Li D, et al. Association between maternal HBsAg carrier status and neonatal adverse outcomes: meta-analysis. J Matern Fetal Neonatal Med. 2015;28:1308-17.

57. Ma X, Sun D, Li C, Ying J, Yan Y. Chronic hepatitis B virus infection and preterm labor (birth) in pregnant women — an updated systematic review and meta-analysis. J Med Virol. 2018;90:93-100.

58. Zhuang $X$, Cui AM, Wang $Q$, Cheng XY, Shen Y, Cai WH, et al. Liver dysfunction during pregnancy and its association with preterm birth in China: a prospective cohort study. EBioMedicine. 2017;26:152-6.

59. Romero R, Gómez R, Chaiworapongsa T, Conoscenti G, Kim JC, Kim YM. The role of infection in preterm labour and delivery. Paediatr Perinat Epidemiol. 2001;15 Suppl 2:41-56.

60. Zeng LN, Zhang LL, Shi J, Gu LL, Grogan W, Gargano MM, et al. The primary microbial pathogens associated with premature rupture of the membranes in China: a systemic review. Taiwan J Obstet Gynecol. 2014;53:443-51.

61. Huang QT, Chen JH, Zhong M, Xu YY, Cai CX, Wei SS, et al. The risk of placental abruption and placenta previa in pregnant women with chronic hepatitis B viral infection: a systematic review and meta-analysis. Placenta. 2014;35:539-45.

62. Gurol-Urganci I, Cromwell DA, Edozien LC, Smith GC, Onwere C, Mahmood TA, et al. Risk of placenta previa in second birth after first birth cesarean section: a population-based study and meta-analysis. BMC Pregnancy Childbirth. 2011;11:95.

63. Pan CQ, Zou HB, Chen Y, Zhang X, Zhang H, Li J, et al. Cesarean section reduces perinatal transmission of hepatitis B virus infection from hepatitis B surface antigen-positive women to their infants. Clin Gastroenterol Hepatol. 2013;11:1349-55.

64. Hu Y, Chen J, Wen J, Xu C, Zhang S, Xu B, et al. Effect of elective cesarean section on the risk of mother-to-child transmission of hepatitis B virus. BMC Pregnancy Childbirth. 2013;13:119.

65. Lao TT, Sahota DS, Cheng YK, Law LW, Leung TY. Advanced maternal age and postpartum hemorrhage-risk factor or red herring? J Matern Fetal Neonatal Med. 2014;27:243-6.

66. Ye F, Liu Y, Jin Y, Shi J, Yang X, Liu X, et al. The effect of hepatitis B virus infected embryos on pregnancy outcome. Eur J Obstet Gynecol Reprod Biol. 2014;172:10-4.

67. Salemi JL, Whiteman VE, August EM, Chandler K, Mbah AK, Salihu HM. Maternal hepatitis B and hepatitis C infection and neonatal neurological outcomes. J Viral Hepat. 2014;21:e144-53. 
68. Lao TT, Sahota DS, Suen SS, Law LW, Leung TY. Maternal HBsAg status and infant size-a Faustian bargain? J Viral Hepat. 2012;19:519-24.

69. Yarrington CD, Cantonwine DE, Seely EW, Mcelrath TF, Zera CA. The association of early unexplained elevated alanine aminotransferase with large-for-gestational-age birthweight. Am J Obstet Gynecol. 2016;215:474.e1-5.

70. Atesci FC, Cetin BC, Oguzhanoglu NK, Karadag F, Turgut H. Psychiatric disorders and functioning in hepatitis B virus carriers. Psychosomatics. 2005;46:142-7.

71. Gupta R, Avasthi A, Chawla YK, Grover S. Psychiatric morbidity, fatigue, stigma and quality of life of patients with hepatitis B infection. J Clin Exp Hepatol. 2020;10:429-41.

72. Zhou F, Li J, Lin K, Ji P, Sun Y. A cross-sectional study on anxiety and stress in pregnant women with chronic HBV infection in the People's Republic of China. Neuropsychiatr Dis Treat. 2015;11:2225-32.

73. Chen X, Chen J, Wen J, Xu C, Zhang S, Zhou YH, et al. Breastfeeding is not a risk factor for mother-to-child transmission of hepatitis B virus. PLoS One. 2013;8:e55303.

74. Shi Z, Yang Y, Wang H, Ma L, Schreiber A, Li X, et al. Breastfeeding of newborns by mothers carrying hepatitis B virus: a meta-analysis and systematic review. Arch Pediatr Adolesc Med. 2011;165:837-46. 